\title{
Effectiveness of Elbasvir and Grazoprevir Combination and 1-year Follow-up Hepatic Function for HCV Genotype 1b Infection: Results From a Chinese Real-world Cohort.
}

Jing Liang

Tianjin Third Central Hospital https://orcid.org/0000-0001-5114-9030

Yaping Zhang

Tianjin Third Central Hospital

Fang Liu

Tianjin Third Central Hospital

Huan Xia

Tianjin City Second People's Hospital

Hongmin Lv

Tianjin Third Central Hospital

Huiling Xiang

Tianjin Third Central Hospital

Yankai Yang

Tianjin Third Central Hospital

Yan Li

Tianjin Third Central Hospital

\section{Tinghong $\mathrm{Li}$}

Tianjin third central hospital

Lei Liu

Tianjin third central hospital

Chengzhen Lu

Tianjin City Second People's Hospital

Fengmei Wang

Tianjin Third Central Hospital

Tao Han ( $\square$ hantaomd@126.com )

Tianjin Third Central Hospital

Research 
Keywords: Chronic hepatitis C cirrhosis, Elbasvir and Grazoprevir, SVR

Posted Date: December 31st, 2020

DOI: https://doi.org/10.21203/rs.3.rs-136726/v1

License: (c) (1) This work is licensed under a Creative Commons Attribution 4.0 International License. Read Full License 


\section{Abstract}

Background: The treatment of chronic hepatitis $\mathrm{C}(\mathrm{CHC})$ has entered the interferon-free era since the approval of all-oral direct-acting antiviral (DAA) therapy in China. Twelve weeks of Elbasvir and Grazoprevir (EBR/GZR) has been demonstrated to be highly effective and well tolerated in phase III registration trials in Asia. However, real-world data on this regimen are lacking in mainland China. We aimed to evaluate the efficacy and safety of EBR/GZR and hepatic function during 1-year follow-up in real-world clinical practice with genotype $1 \mathrm{~b}$ HCV infection.

Methods: A prospective, multicenter, non-interventional cohort study was conducted in Tianjin Third Central Hospital and Tianjin Second People's Hospital of China. All patients diagnosed with HCV-1b infection and treated with EBR/GZR for 12 weeks were included. The sustained virological response (SVR) rate obtained 12 weeks posttreatment (SVR12) and week 48 posttreatment (SVR48), the liver function and safety were also evaluated in patients who received EBR/GZR.

Results: A total of 251 patients were enrolled, 215 of whom completed the 12-week treatment and 107 completed the 48-week follow-up after treatment. 47 patients (21.9\%) had compensatory cirrhosis. The overall rates at the end of treatment (EOT), SVR12 and SVR48 were $100 \%, 99.5 \%$ and $99.1 \%$, respectively. Two cirrhosis patients relapsed at 12 weeks and 48 weeks posttreatment respectively. At 48 weeks posttreatment, the normalization rate of ALT and AST were increased significantly than that of baseline ( $97.2 \%$ and $95.3 \%$ vs $59.5 \%$ and $61.4 \%, P<0.01$ ). The incidence of any adverse reactions was $18.1 \%$, while most reactions were mild in severity.

Conclusion: In a real-world cohort, treatment with EBR/GZR in Chinese patients with genotype 1b HCV infection appears to be well tolerated and achieves high SVR12 and SVR48 rates.

Trial registration: ChiCTR, ChiCTR1900023747. Registered 10 June 2019, Retrospectively registered. http://www.chictr.org.cn/showproj.aspx?proj=39926

\section{Introduction}

Chronic hepatitis $\mathrm{C}(\mathrm{CHC})$ is a chronic, progressive disease that occurs worldwide and greatly threatens human health[1, 2]. In China, hepatitis $C$ virus infection is one of the most important causes of cirrhosis and hepatocellular carcinoma[3]. Because of the excellent efficacy and limited course of direct-acting antiviral drugs (DAA), interferon-free oral antiviral drugs have been recommended as first-line antiviral therapy for chronic hepatitis $\mathrm{C}$ patients[4]. With the introduction of direct-acting antiviral drugs in China, hepatitis $C$ treatment has progressed from interferon to the DAA era.

Genotype $1 \mathrm{~b}$ HCV accounts for $62.7 \%$ of HCV infections in China[5]. Combination therapy with Elbasvir (EBR) / Grazoprevir (GZR) has been demonstrated to be safe and highly effective for genotype-1 hepatitis $\mathrm{C}$ virus (HCV)-infected patients in phase III registration trials in Asia[6] and approved for use in Chinese patients with hepatitis C since November 2018. However, the real-world data regarding this regimen in 
Chinese patients are not well known. We aimed to evaluate the efficacy and safety of Elbasvir/Grazoprevir for the treatment of Chinese patients with chronic HCV genotype $1 \mathrm{~b}$ infection in the 1-year follow-up.

\section{Patients And Methods Study population}

In this prospective, multicenter, noninterventional cohort study, patients with genotype $1 \mathrm{~b}$ chronic hepatitis $\mathrm{C}$ and compensated cirrhosis who received a 12-week treatment course with EBR / GZR from November 1, 2018, to August 31, 2019, in Tianjin Third Central Hospital and Tianjin 2nd People's Hospital were continuously enrolled.

Adult patients ( $>18$ years old) diagnosed with chronic HCV-1 infection and treated with EBR /GZR were eligible for this study. Chronic hepatitis $C$ virus infection, defined as detectable hepatitis $C$ virus antibodies and quantifiable serum HCV RNA by using the COBAS TaqMan HCV Kit (Roche Diagnosis Co, Ltd, Mannheim, Germany, detection limit [LLOD]: $15 \mathrm{IJ} / \mathrm{ml}$ ), lasted for more than six months. The diagnosis of liver cirrhosis was based on transient elastography exceeding $12.5 \mathrm{kPa}[7]$ and a liver imaging examination (ultrasonography, computed tomography or MR imaging) that revealed signs of cirrhosis (e.g., atrophy of the right lobe of the liver, irregularity of the hepatic surface, and splenomegaly) in combination with the clinical state. The exclusion criteria were as follows: decompensated cirrhosis, coinfection with HBV or HIV, self-withdrawal during treatment due to non-drug reasons, treatment duration less or more than 12 weeks, incomplete data, loss to follow-up or previous history of DAA drug use.

Patients enrolled in the study received EBR $(100 \mathrm{mg})$ and GZR $(50 \mathrm{mg})$ once daily without ribavirin for 12 weeks. The clinical, laboratory and virological parameters were assessed at baseline, end of treatment, 12 weeks and 48 weeks after treatment completion. HCV RNA was detected using a Roche COBAS HCV Kit with a range of $15 \sim 6.9 * 10^{7} \mathrm{IU} / \mathrm{ml}$. This observational study was approved by the Ethics Committee of Tianjin Third Central Hospital and Tianjin 2nd People's Hospital. Informed consent was obtained from all the patients prior to study initiation.

\section{Evaluations of efficacy and safety}

Virologic response, defined as undetectable HCV RNA, was assessed at the end of treatment (EOT), at week 12 (SVR12) posttreatment and week 48 (SVR 48) posttreatment. Improvements in liver function were also assessed at the same time.

Any adverse events (AEs), including clinical and biochemical abnormalities associated with treatment reported by patients during treatment, were recorded. The MedDRA system is used to code adverse events and associated diseases[8]. Details of all recorded serious adverse events (SAEs) were collected by the treating physicians. SAEs were defined as any life-threatening event, an event that led to hospital admission, or one that resulted in death. 


\section{Statistics}

All the statistical analyses were performed using SPSS software, version 22.0 (IBM Corporation, Somers, NY, USA). Quantitative variables are presented as and range when appropriate. The viral responses are shown in numbers and percentages with $95 \%$ confidence intervals (Cls). A two-sided p-value of $<0.05$ was considered statistically significant. The statistical analysis was performed.

\section{Results}

\section{Baseline Characteristics of Patients}

From November 1, 2018, to August 31, 2019, 468 HCV-1 infection patients were enrolled in this observational cohort study in the two medical centers. A total of 215 patients met the admission criteria and completed the course of treatment and 12-week' follow-up,107 patients completed 48-week' followup by the end of Nov 2020, shown in Fig. 1. Of these 215 patients with an average age of 60 years, 8 patients had previously been treated with interferon and ribavirin before DAA treatment. Among the baseline population, $21.9 \%$ had compensated cirrhosis. 9 patients with uremia who underwent dialysis were also enrolled in our study. The demographic and baseline clinical characteristics of the 215 patients are shown in Table 1. 
Table 1

Baseline characteristics of patients treated with EBR/GZR

\begin{tabular}{|ll|}
\hline Characteristics & Patients $(\mathrm{N}=215)$ \\
\hline Sex, male, $\mathrm{n}(\%)$ & $91(42.1)$ \\
\hline Age, years, median (range) & $60(28-87)$ \\
\hline Treatment-naïve, $\mathrm{n},(\%)$ & $207(96.3)$ \\
\hline Cirrhosis, $\mathrm{n},(\%)$ & $47(21.9)$ \\
\hline On dialysis, $\mathrm{n},(\%)$ & $9(4.2)$ \\
\hline History of HCC, $\mathrm{n},(\%)$ & $1(0.5)$ \\
\hline HCV RNA, log $10 \mathrm{IU} / \mathrm{mL}$, median (range) & $6.4(2.0-8.7)$ \\
\hline ALT, U/L, median (range) & $36.0(3.0-819.0)$ \\
\hline AST, U/L, median (range) & $35.0(11.0-705.0)$ \\
\hline Total bilirubin, $\mu$ mol/L, median (range) & $14.5(5.4-57.0)$ \\
\hline Albumin, g/L, median (range) & $44.6(23.4-55.4)$ \\
\hline PLT, $\times 10^{9} / \mathrm{L}$, median (range) & $148.0(11.0-410.0)$ \\
\hline a-Fetoprotein, ng/mL, median (range) & $5.2(0.6-193.2)$ \\
\hline Transient elastography (FibroScan), kPa, median (range) & $9.1(3.3-51.4)$ \\
\hline Creatinine $\mu$ mol/L, median (range) & $65.0(38.9-1058)$ \\
\hline
\end{tabular}

\section{Virological response}

At EOT,12 weeks and 48 weeks posttreatment, the proportions of all patients with undetectable HCV RNA were 100\% (215/215), 99.5\% (214/215) and 99.1\% (106/107), respectively (Fig. 2). One cirrhosis patient relapsed 12 weeks after treatment with HCV RNA of $3.66^{*} 10^{\wedge} 5 \mathrm{IU} / \mathrm{ml}$, who had been discontinuing medication for 6 weeks. The overall SVR12 rate was $97.9 \%$ (46/47) in patients with liver cirrhosis and $100 \%$ in non-cirrhosis patients. By November 30, 2020, a total of 107 patients obtained 48-week follow-up data. One patient with liver cirrhosis recurred 48 weeks after treatment with HCV RNA of $1.00^{*} 10^{\wedge} 6 \mathrm{IU} / \mathrm{ml}$. She was a non-reinfected patient with the same genotype as the baseline. Virological responses were obtained in all 9 uremic and dialysis patients 12 weeks and 48 weeks after treatment.

\section{Biochemical changes}

The ALT, AST, TBIL and Albumin levels and normalization rate of all patients at baseline, EOT,12 and 48 weeks posttreatment are shown in Table 2. The ALT and AST normalization rate with EBR/GZR treatment 
increased significantly at EOT,12 weeks and 48 weeks posttreatment compared with the baseline (ALT $91.6 \%, 96.3 \%, 97.2 \%$ vs $59.5 \%$, P $₫ 0.01$; AST $85.6 \%, 95.3 \%, 95.3 \%$ vs $61.4 \%, P \llbracket 0.01)$. At EOT,12 weeks and 48 weeks posttreatment, serum creatinine levels had no significant change compared with the baseline (66.0,65.0,67.0 vs 65.0umol/I, $\mathrm{P}>0.05)$. During the 48-week follow-up, no patients developed decompensated cirrhosis.

Table 2

Levels and normalization rates of ALT, AST and TBIL at baseline, EOT,12 weeks posttreatment and 48w posttreatment

\begin{tabular}{|c|c|c|c|c|}
\hline Liver function parameters & Baseline & EOT & $\begin{array}{l}12 \mathrm{w} \\
\text { Posttreatment }\end{array}$ & $\begin{array}{l}48 \mathrm{w} \\
\text { Posttreatment }\end{array}$ \\
\hline Patients N & 215 & 215 & 215 & 107 \\
\hline ALT, U/L, median (range) & $\begin{array}{l}36.0 \\
(3.0-819.0)\end{array}$ & $\begin{array}{l}17.0 \\
(2.0-111.0)\end{array}$ & $\begin{array}{l}16.0 \\
(3.0-142.0)\end{array}$ & $\begin{array}{l}15.0 \\
(4.0-105.0)\end{array}$ \\
\hline ALT Normalization rate, $\mathrm{n} / \mathrm{N}(\%)$ & $\begin{array}{l}128 / 215 \\
(59.5 \%)\end{array}$ & $\begin{array}{l}197 / 215^{\triangle} \\
(91.6 \%)\end{array}$ & $\begin{array}{l}207 / 215^{\triangle} \\
(96.3 \%)\end{array}$ & $\begin{array}{l}104 / 107^{\triangle} \\
(97.2 \%)\end{array}$ \\
\hline AST, U/L, median (range) & $\begin{array}{l}35.0 \\
(11.0-705.0)\end{array}$ & $\begin{array}{l}11.0 \\
(8.0-76.0)\end{array}$ & $\begin{array}{l}20.0 \\
(5.0-95.0)\end{array}$ & $\begin{array}{l}21.0 \\
(9.0-107.0)\end{array}$ \\
\hline AST Normalization rate, n/N (\%) & $\begin{array}{l}132 / 215 \\
(61.4 \%)\end{array}$ & $\begin{array}{l}184 / 215^{\triangle} \\
(85.6 \%)\end{array}$ & $\begin{array}{l}205 / 215^{\triangle} \\
(95.3 \%)\end{array}$ & $\begin{array}{l}102 / 107^{\triangle} \\
(95.3 \%)\end{array}$ \\
\hline TBIL, $\mu$ mol/L, median (range) & $\begin{array}{l}14.5 \\
(5.4-57.0)\end{array}$ & $\begin{array}{l}13.6 \\
(4.0-59.0)\end{array}$ & $\begin{array}{l}13.7 \\
(3.2-54.0)\end{array}$ & $\begin{array}{l}13.9 \\
(4.8-48.8)\end{array}$ \\
\hline TBIL Normalization rate, $\mathrm{n} / \mathrm{N}(\%)$ & $\begin{array}{l}147 / 215 \\
(68.4 \%)\end{array}$ & $\begin{array}{l}160 / 215 \\
(74.4 \%)\end{array}$ & $\begin{array}{l}162 / 215 \\
(75.3 \%)\end{array}$ & $\begin{array}{l}78 / 107 \\
(72.9 \%)\end{array}$ \\
\hline Albumin, $g / L$, median (range) & $\begin{array}{l}44.6 \\
(23.4-55.4)\end{array}$ & $\begin{array}{l}46.3 \\
(31.7-53.7)\end{array}$ & $\begin{array}{l}46.6 \\
(32.9-52.6)\end{array}$ & $\begin{array}{l}46.8 \\
(30.1-52.6)\end{array}$ \\
\hline ALB Normalization rate, $\mathrm{n} / \mathrm{N}(\%)$ & $\begin{array}{l}199 / 215 \\
(92.5 \%)\end{array}$ & $\begin{array}{l}212 / 215 \\
(98.6 \%)\end{array}$ & $\begin{array}{l}214 / 215 \\
(99.5 \%)\end{array}$ & $\begin{array}{l}106 / 107 \\
(99.1 \%)\end{array}$ \\
\hline $\begin{array}{l}\text { Creatinine } \mu \mathrm{mol} / \mathrm{L} \text {, median } \\
\text { (range) }\end{array}$ & $\begin{array}{l}65.0 \\
(38.9- \\
1058)\end{array}$ & $\begin{array}{l}66.0 \\
\left(36.0^{-}\right. \\
1134.0)\end{array}$ & $\begin{array}{l}65.0 \\
(34.0-1144)\end{array}$ & $\begin{array}{l}67.0 \\
(40.0-1031.0)\end{array}$ \\
\hline$\triangle \mathrm{P}<0.01$ & & & & \\
\hline
\end{tabular}




\section{Safety and adverse events}

In general, our results indicated that the safety of EBR/GZR treatment in HCV-1 patients was tolerable. The overall incidence of AEs was $18.1 \%(n=39)$, which was mild to moderate. One patient was interrupted for 6 weeks due to non-drug factors during treatment. The most frequent adverse events were elevated total bilirubin (7.4\%), fatigue (4.7\%), dizziness (2.3\%) and nausea (2.3\%), see Table 3. Severe adverse events and death did not occur in our study.

Table 3

Adverse events occurring during treatment of patients treated with EBR/GZR

\begin{tabular}{|ll|}
\hline Adverse events & Patients, $\mathbf{n}(\%) \mathbf{~}=\mathbf{2 1 5}$ \\
\hline Interruption during treatment & $1(0.5)$ \\
\hline Any adverse event & $39(18.1)$ \\
\hline Elevated total bilirubin & $16(7.4)$ \\
\hline Fatigue & $10(4.7)$ \\
\hline Dizziness & $5(2.3)$ \\
\hline Nausea & $5(2.3)$ \\
\hline Itchy skin & $2(0.9)$ \\
\hline Weight loss & $2(0.9)$ \\
\hline Anemia & $1(0.5)$ \\
\hline Serious adverse events & $0(0.0)$ \\
\hline Death & $0(0.0)$ \\
\hline
\end{tabular}

\section{Discussion}

Rapid improvements in hepatitis $\mathrm{C}$ virus (HCV) therapy have significantly increased the cure rate of chronic hepatitis $C[9,10]$. However, this therapy truly entered the DAA era with its approval in Apr 2018 in China. Epidemiological studies reported that HCV genotype 1 infection was the most prevalent genotype in the world and China[11-13]. As a powerful antiviral regimen for HCV-1b has been demonstrated in clinical studies, there are several reports on the outcomes of EBR /GZR treatment in real-world clinical practice[14-16]; however, there are few reports from the Chinese mainland. Chinses patients in the real world are more heterogeneous and have more complications comparing with those in RCT studies, and there are differences in viral infection pathways, races from other countries such as Europe and the United States, therefore, it is worthwhile for us to discuss the real effect of this regimen on hepatitis $\mathrm{C}$ patients in China. This real-world cohort study from the Chinese mainland was aimed to observe the 
efficacy and safety of the EBR / GZR for hepatitis C patients, and the liver function in the 1-year follow-up period after treatment.

Through the inclusion of compensatory cirrhosis patients, our study showed that the overall SVR12 rate in patients receiving EBR / GZR was excellent (99.5\%) and was slightly higher than the response rates(98.2\%)in the clinical trials from the Asia-Pacific region and Russia[6]. Of the 47compensated cirrhotic patients in our study, $100 \%$ of patients achieved a virological response at the end of treatment, and 46 patients $(97.6 \%)$ achieved SVR12, which was similar to the previous real-world studies[14, 17, 18]. Although SVR12 was considered to be hepatitis C virus cure[19], in our study, one cirrhosis patient relapsed 48 weeks posttreatment after receiving SVR12. Long term follow-up of patients with liver cirrhosis after HCV treatment was still necessary.

During the treatment, liver function improved significantly and more than $91 \%$ of patients had normal ALT levels at the end of treatment. At 48 weeks posttreatment, $97.2 \%$ of patients' ALT levels and $95.3 \%$ of patients' AST levels remained normal. We found that overall liver function was relatively stable and there was no progression of decompensated cirrhosis during the 48-week follow-up.

In this study, 9 patients with chronic renal failure and regular dialysis completed 12 weeks of antiviral treatment with EBR /GZR without drug reduction. Previous studies on patients with advanced kidney disease have suggested that EBR /GZR is well tolerated[20]. All 9 patients achieved SVR12 and SVR48 in our study which was consistent with our previous findings[21], indicating that this regimen had good therapeutic effect and tolerance in renal failure patients.

Our data showed the safety and tolerability (including renal safety) of EBR /GZR. One patient discontinued treatment for 6 weeks because of his original disease, while no patient discontinued because of drug interactions. In this study, side effects were reported in 39 patients. Although the reported $A E$ incidence in this study was only slightly higher than that in some real-world studies $[15,16]$, most of AEs were mild in severity. During treatment, the hyperbilirubinemia observed was the most common adverse event; however, the severity was mainly graded 1 or 2 and lasted only 2-4 weeks for most patients. In general, EBR / GZR was well tolerated and safe in clinical practice.

Since DAA in China has been available in the past year and is expensive, there are few large sample reports about the real-world study. Although this study reflects the real-world data of HCV-1b patients, several limitations existed in our study. First, the patients in this study came from two medical centers, while they were limited in one region and the sample size was small. Second, there was potential bias in the doctor prescriptions and incomplete patient records in the real-world efficacy evaluation. Moreover, our study included patients with chronic hepatitis $\mathrm{C}$ and compensatory cirrhosis and lacked data on a large sample of cirrhotic patients.

\section{Conclusion}


In conclusion, in this real-world study, 12-week Elbasvir / Grazoprevir was shown to achieve a high SVR12 of $99.5 \%$ in Chinese patients with genotype- 1 b hepatitis $C$ and maintain $99.1 \%$ at 48 weeks posttreatment. Liver function improved after treatment and during the 48 weeks posttreatment. Elbasvir /Grazoprevir therapy, with excellent efficacy and tolerance, is an effective therapeutic option for patients with chronic hepatitis $\mathrm{C}$ and compensatory cirrhosis.

\section{Abbreviations}

CHC chronic hepatitis C; DAA direct-acting antiviral drug; EBR Elbasvir; GZR Grazoprevir; HCV hepatitis C virus; SVR sustained virologic response; EOT end of treatment; AEs adverse events; SAEs serious adverse events; ALT alanine aminotransferase; AST aspartate aminotransferase; TBIL total bilirubin; ALB Albumin

\section{Declarations}

\section{Ethics approval and consent to participate}

This study was approved by the Ethics Committee of Tianjin Third Central Hospital and informed consent was obtained from all patients prior to study initiation.

\section{Consent for publication}

Not applicable

\section{Availability of data and materials}

All data generated or analyzed during this study are included in this published article.

\section{Competing interests}

The authors declare that they have no competing interests

\section{Funding}

The study was supported by the National 13th 5-year Plan for Hepatitis Research (2017ZX10203201-007) and the National Natural Science Foundation of China (No.81870429『

\section{Authors's contributions}

LJ and ZYP contributed equally to this work. LJ, WFM and TH designed the research. LJ and ZYP drafted the manuscript. LJ and ZYP collected the data and established the database. YYC and FL presided over the enrollment and exclusion of the research subjects. LF, XH, LHM, XHL, YYK, LTH, LL and LCZ managed and followed up the patients. LJ analyzed the data statistically. WFM and TH participated in paper modification and revised the manuscript for English writing. All authors read and approved the final manuscript. 
Acknowledgements

Not applicable

\section{References}

1. Global prevalence and genotype distribution of hepatitis $C$ virus infection in 2015: a modelling study. Lancet Gastroenterol Hepatol 2017, 2:161-176.

2. Teshale EH, Xing J, Moorman A, Holmberg SD, Spradling PR, Gordon SC, Rupp LB, Lu M, Boscarino JA, Trinacity CM, et al: Higher all-cause hospitalization among patients with chronic hepatitis C: the Chronic Hepatitis Cohort Study (CHeCS), 2006-2013. J Viral Hepat 2016, 23:748-754.

3. Pan YF, Zheng Y, Qin T, Feng L, Zhang Q, Ping XG, Pan YT, Wang XP, Bai L, Li HH: Disease progression in Chinese patients with hepatitis $\mathrm{C}$ virus RNA-positive infection via blood transfusion. Exp Ther Med 2016, 12:3476-3484.

4. EASL Recommendations on Treatment of Hepatitis C 2016. J Hepatol 2017, 66:153-194.

5. Zhang Y, Chen LM, He M: Hepatitis C Virus in mainland China with an emphasis on genotype and subtype distribution. Virol J 2017, 14:41.

6. Wei L, Jia JD, Wang FS, Niu JQ, Zhao XM, Mu S, Liang LW, Wang Z, Hwang P, Robertson MN, et al: Efficacy and safety of elbasvir/grazoprevir in participants with hepatitis $C$ virus genotype 1, 4, or 6 infection from the Asia-Pacific region and Russia: Final results from the randomized C-CORAL study. J Gastroenterol Hepatol 2019, 34:12-21.

7. Cardoso AC, Carvalho-Filho RJ, Stern C, Dipumpo A, Giuily N, Ripault MP, Asselah T, Boyer N, Lada O, Castelnau C, et al: Direct comparison of diagnostic performance of transient elastography in patients with chronic hepatitis B and chronic hepatitis C. Liver Int 2012, 32:612-621.

8. Brown EG: Methods and pitfalls in searching drug safety databases utilising the Medical Dictionary for Regulatory Activities (MedDRA). Drug Saf 2003, 26:145-158.

9. Afdhal N, Zeuzem S, Kwo P, Chojkier M, Gitlin N, Puoti M, Romero-Gomez M, Zarski JP, Agarwal K, Buggisch P, et al: Ledipasvir and sofosbuvir for untreated HCV genotype 1 infection. N Engl J Med 2014, 370:1889-1898.

10. Ji F, Yeo YH, Wei MT, Ogawa E, Enomoto M, Lee DH, lio E, Lubel J, Wang W, Wei B, et al: Sustained virologic response to direct-acting antiviral therapy in patients with chronic hepatitis $\mathrm{C}$ and hepatocellular carcinoma: A systematic review and meta-analysis. J Hepatol 2019.

11. Chen Y, Yu C, Yin X, Guo X, Wu S, Hou J: Hepatitis C virus genotypes and subtypes circulating in Mainland China. Emerg Microbes Infect 2017, 6:e95.

12. Yuan G, Liu J, Hu C, Huang H, Qi M, Wu T, Liang W, Li YP, Zhang YY, Zhou Y: Genotype Distribution and Molecular Epidemiology of Hepatitis C Virus in Guangzhou, China: Predominance of Genotype 1b and Increasing Incidence of Genotype 6a. Cell Physiol Biochem 2017, 43:775-787. 
13. Messina JP, Humphreys I, Flaxman A, Brown A, Cooke GS, Pybus OG, Barnes E: Global distribution and prevalence of hepatitis $C$ virus genotypes. Hepatology 2015, 61:77-87.

14. Puenpatom A, Cao Y, Yu X, Kanwal F, El-Serag HB, Kramer JR: Effectiveness of Elbasvir/Grazoprevir in US Veterans with Chronic Hepatitis C Virus Genotype 1b Infection. Infect Dis Ther 2020, 9:355-365.

15. Mashiba T, Joko K, Kurosaki M, Ochi H, Hasebe C, Akahane T, Sohda T, Tsuji K, Mitsuda A, Kimura H, et al: Real-world efficacy of elbasvir and grazoprevir for hepatitis $C$ virus (genotype 1): A nationwide, multicenter study by the Japanese Red Cross Hospital Liver Study Group. Hepatol Res 2019, 49:1114-1120.

16. Hernandez-Conde M, Fernandez I, Perello C, Gallego A, Bonacci M, Pascasio JM, Romero-Gomez M, Llerena S, Fernandez-Rodriguez C, Castro Urda JL, et al: Effectiveness and safety of elbasvir/grazoprevir therapy in patients with chronic HCV infection: Results from the Spanish HEPA-C real-world cohort. J Viral Hepat 2019, 26:55-64.

17. Tsai TC, Deng ST, Hsu CW: The efficacy and safety of elbasvir/grazoprevir treatment in HCV genotype 1 patients in Taiwan. J Med Virol 2020, 92:219-226.

18. Jang ES, Kim KA, Kim YS, Kim IH, Lee BS, Lee YJ, Chung WJ, Jeong SH: Effectiveness and safety of elbasvir/grazoprevir in Korean patients with hepatitis $\mathrm{C}$ virus infection: a nationwide real-world study. Korean J Intern Med 2020.

19. Sung JC, Bosh C, Wyatt B, Miller M, Harty A, Del Bello D, Knight S, Dieterich DT, Perumalswami PV, Branch AD: Hepatitis $C$ cure improved patient-reported outcomes in patients with and without liver fibrosis in a prospective study at a large urban medical center. $J$ Viral Hepat 2020, 27:350-359.

20. Roth D, Nelson DR, Bruchfeld A, Liapakis A, Silva M, Monsour H, Jr., Martin P, Pol S, Londono MC, Hassanein T, et al: Grazoprevir plus elbasvir in treatment-naive and treatment-experienced patients with hepatitis $C$ virus genotype 1 infection and stage 4-5 chronic kidney disease (the C-SURFER study): a combination phase 3 study. Lancet 2015, 386:1537-1545.

21. Li C, Liang J, Xiang $\mathrm{H}$, Chen $\mathrm{H}$, Tian J: Effectiveness of direct-acting antivirals in maintenance hemodialysis patients complicated with chronic hepatitis C. Medicine (Baltimore) 2020, 99:e23384.

\section{Figures}


Reasons for exclusion:

-Other DAA regimens $(n=217)$

\section{$468 \mathrm{HCV}-1 \mathrm{~b}$ infected}

patients received DAAs

reqimens

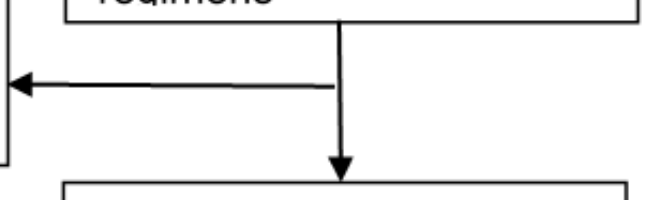

251 patients received

EBR /GZR regimen for 12 weeks
Reasons for exclusion:

-Co-infection with HBV ( $n=1)$

-Incomplete data $(n=20)$

-Lost to follow-up ( $n=15)$
215 patients completed $12-$ week treatment and 12week follow-up were enrolled

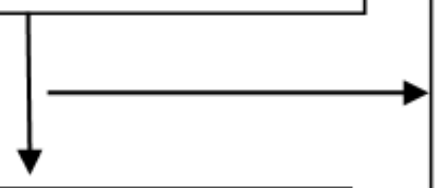

107 patients completed 48week follow-up
Reasons for exclusion:

-Less than 48 weeks follow-up $(n=66)$ -Incomplete data $(n=15)$ -Lost to follow-up $(n=27)$

\section{Figure 1}

Patient recruitment flowchart 


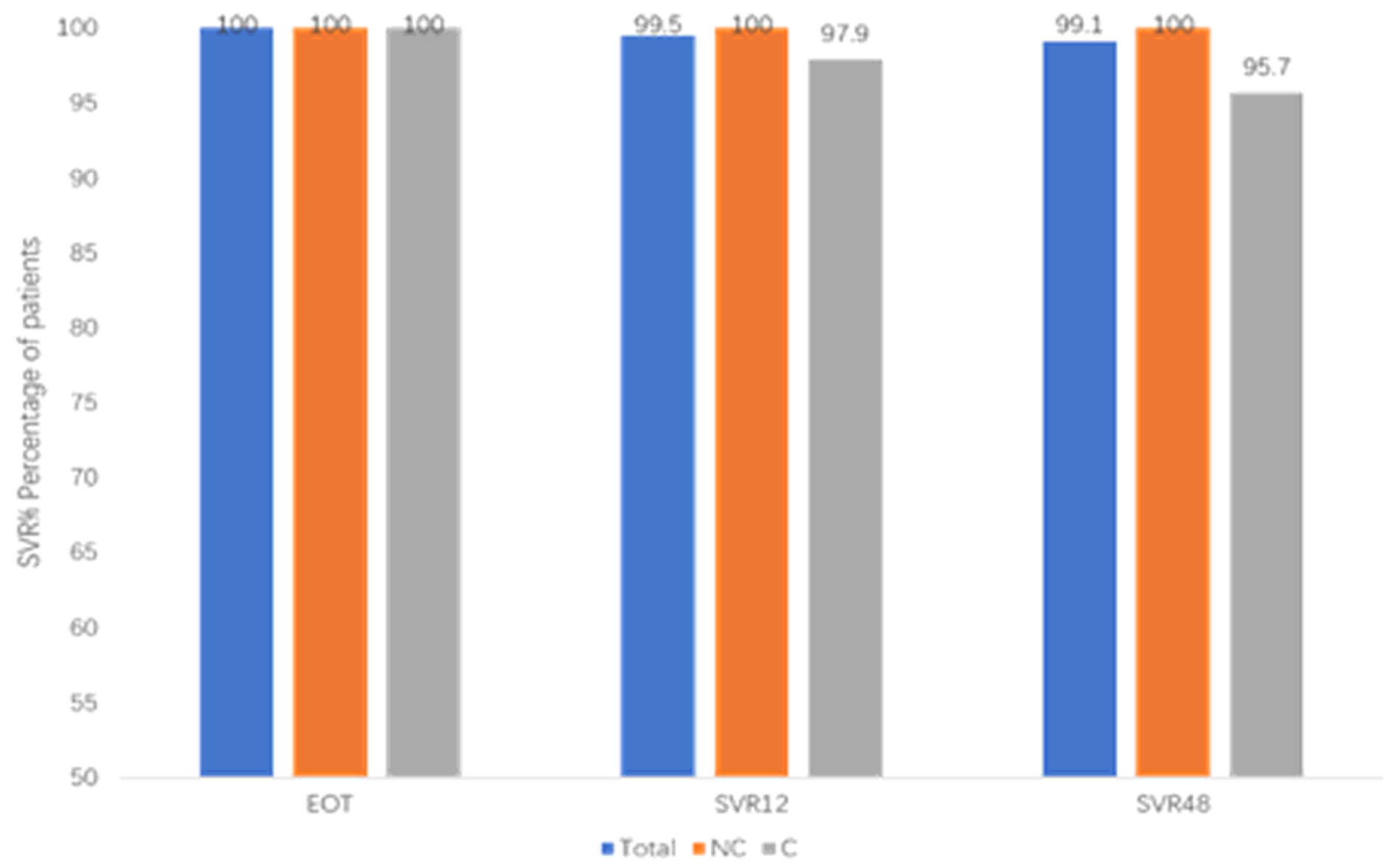

Figure 2

Percentage of patients with HCV RNA $<$ Lower Limit of Detection 